CASE REPORT

\author{
R. Kuo \\ J.K. Huang \\ K.S. Lee \\ B.F. Chen \\ F.S. Yang
}

\title{
Leiomyosarcoma in the Nasopharynx: MR Imaging Findings
}

SUMMARY: The MR imaging findings of a leiomyosarcoma arising from the nasopharynx are presented. To our knowledge, this is the first MR imaging description of this entity.

eiomyosarcomas are uncommon malignant mesenchymal -tumors that seldom occur in the sinonasal tract. Most of the previously reported cases have occurred in the paranasal sinuses and nasal cavity. ${ }^{1,2}$ Leiomyosarcoma arising from the nasopharynx is extremely unusual. We describe the imaging findings in such a case.

\section{Case Report}

A 23-year-old man presented with nasal obstruction for 2 months with occasional epistaxis. Nasopharyngeal examination revealed a large bulging mass with a normal-appearing mucosa in the nasopharynx. There was no palpable cervical or supraclavicular lymphadenopathy. The patient was HIV-negative.

MR imaging revealed a well-demarcated tumor arising from the posterior wall of the nasopharynx extending inferiorly to the oropharynx (Fig 1A). The tumor was confined within the nasopharynx and oropharynx without involvement of sphenopalatine foramen and pterygopalatine fossa on either side (Fig $1 B$ ). The mass had intermediate signal intensity on T1-weighted images (Fig $1 C$ ) and high signal intensity on T2-short $\tau$ inversion recovery (STIR) images (Fig $1 D$ ), with strong homogeneous enhancement (Fig 1E). There was a mass effect on the adjacent longus capitis muscles (Fig 1C) with well-preserved lateral pharyngeal recesses bilaterally (Fig $1 F)$. A 1 -cm enlarged lymph node was seen in the right lateral retropharyngeal space (Fig $1 D)$. No cervical lymphadenopathy could be identified.

Surgery was performed through a transpalatal approach. A solid tumor mass arising from the posterior wall of the nasopharynx with extension to the oropharynx was found, consistent with the location on imaging. It was successfully removed with wide local excision.

The pathologic findings of the tumor were consistent with a moderately differentiated leiomyosarcoma, including features of a spindle cell sarcoma (Fig 2A). The tumor cells were pleomorphic, with atypical mitoses (Fig $2 B$ ) and a strong immunoreactivity for desmin (Fig $2 C)$. Immunohistochemical stains for cytokeratin and S-100 protein were negative.

\section{Discussion}

Leiomyosarcoma is an uncommon malignant smooth muscle tumor that is most often found in the gastrointestinal tract,

Received November 1, 2006; accepted after revision December 1.

From the Department of Radiology (R.K., J.K.H., F.S.Y.), Mackay Memorial Hospital, Taipei, Taiwan; Taipei Medical University (J.K.H.), Taipei, Taiwan; and the Departments of Otolaryngology (K.S.L.) and Pathology (B.F.C.), Mackay Memorial Hospital, Taipei, Taiwan.

Please address correspondence to Jon-Kway Huang, MD, Department of Radiology, Mackay Memorial Hospital, 92, Section 2, Chungshan N Rd, Taipei, Taiwan; e-mail: kuoricha@ms1.mmh.org.tw

DOI 10.3174/ajnr.A0560 uterus, and retroperitoneum. It constitutes approximately $6.5 \%$ of all soft-tissue sarcomas and only $1.5 \%-2.3 \%$ arising in the sinonasal tract. ${ }^{2}$ Leiomyosarcoma arising from the nasopharynx is even more unusual. To our knowledge, it has been reported only 4 times in the past. ${ }^{3-6}$ In the sinonasal tract, leiomyosarcoma is believed to arise from the wall of blood vessels because these are the only structures containing smooth muscle in this anatomic area. ${ }^{7}$

Our initial differential diagnosis for the patient's imaging findings included nasopharyngeal cancer, juvenile angiofibroma, lymphoid hyperplasia, lymphoma, benign mixed tumor, or malignancy of the minor salivary gland in the nasopharynx.

Nasopharyngeal cancer is the most common nasopharyngeal malignancy among Chinese. It is most often centered in the lateral pharyngeal recess of the nasopharynx and frequently invades the adjacent deep fascial spaces. The smooth margin and absence of lateral pharyngeal recess involvement in our patient would be unusual for a nasopharyngeal cancer. Also, most nasopharyngeal cancers do not have strong homogeneous enhancement such as we saw in the present patient.

Given the patient's sex and age and the strong enhancement of the tumor, one might think of juvenile angiofibroma. However, this tumor almost always originates from the sphenopalatine foramen with early penetration of the pterygopalatine fossa. Absence of sphenopalatine foramen involvement in this patient made juvenile angiofibroma unlikely. Lymphoid hyperplasia is characterized by internal enhancing septa on gadolinium-enhanced T1-weighted imaging, which was absent in this patient. Lymphoma may arise from the adenoids in the nasopharynx. It often shows low-grade enhancement and is usually associated with large cervical lymphadenopathy.

Benign mixed tumors of the minor salivary glands in the nasopharynx often present as well-demarcated submucosal tumors, which may be pedunculated if particularly large. However, the presence of an adjacent enlarged right lateral retropharyngeal lymph node in our patient made a benign cause less likely. Minor salivary gland malignancy in the nasopharynx usually presents as an infiltrative mass with perifocal extension. Occasionally, though, it appears as a pedunculated mass with a smooth margin and thus could not be ruled out in this patient by the imaging features alone.

The prognosis of sinonasal leiomyosarcomas is poor. Although there is a case report that etoposide and high-dose ifosfamide caused significant tumor shrinkage, ${ }^{8}$ chemotherapy and radiation therapy are generally considered to be ineffective in the management of sinonasal tract leiomyosarco- 

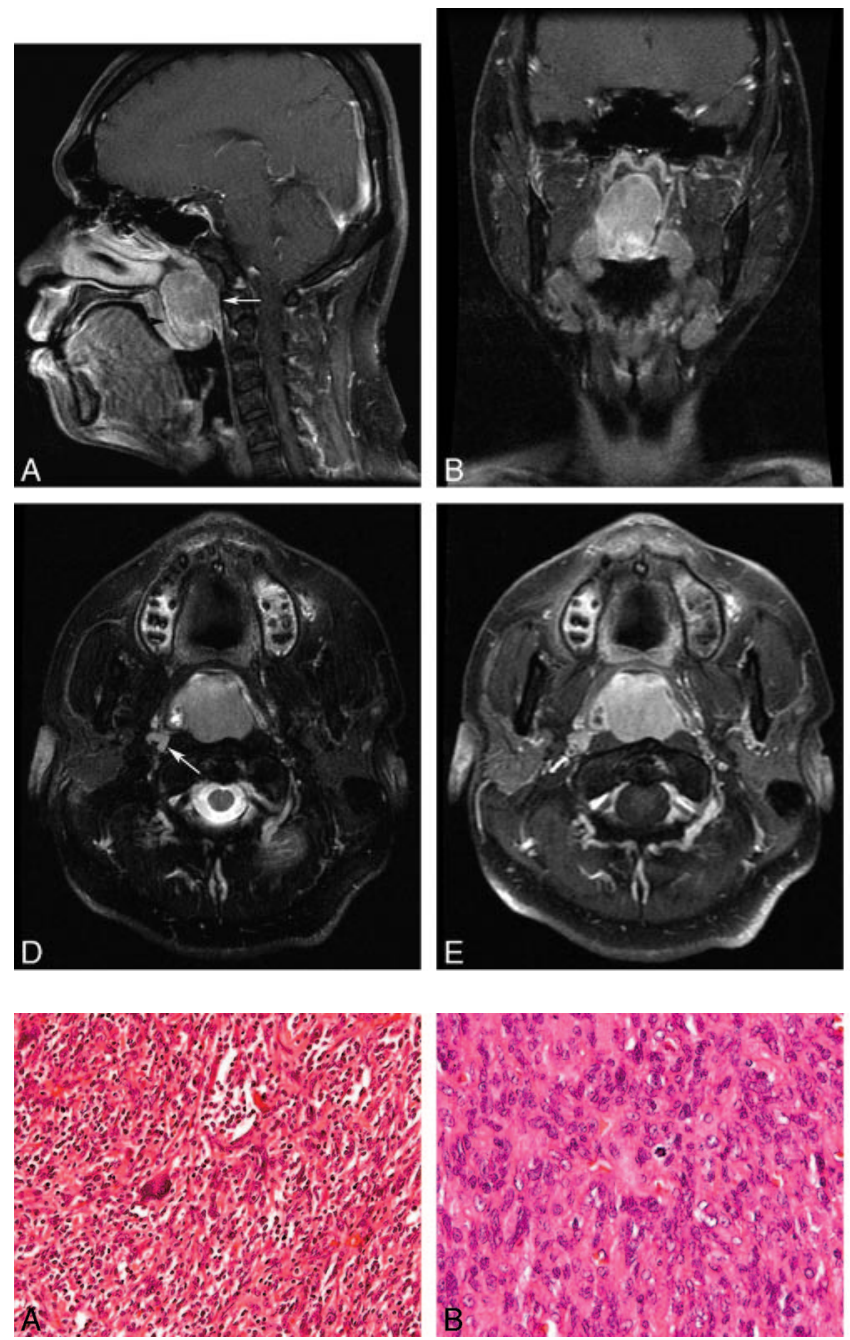

mas. Wide local excision is the treatment of choice because the tumor is characterized as locally aggressive rather than as having high metastatic potential. ${ }^{9}$ Precise identification of the extent of tumor involvement and subsequent complete surgical resection are essential.

\section{Conclusion}

When a well-demarcated strongly enhancing tumor arising from the nasopharynx with a mass effect on the adjacent structures is demonstrated on MR imaging, leiomyosarcoma should be included in the differential diagnosis. Accurate delineation of the extent of the tumor is crucial to optimal management.

\section{References}

1. Huang HY, Antonescu CR. Sinonasal smooth muscle cell tumors: a clinicopathologic and immunohistochemical analysis of 12 cases with emphasis on the low-grade end of the spectrum. Arch Pathol Lab Med 2003;127:297-304 genol 1994;163:425-32 1958;68:211-13 1996;58:115-20 Surg 1990;116:1278-86
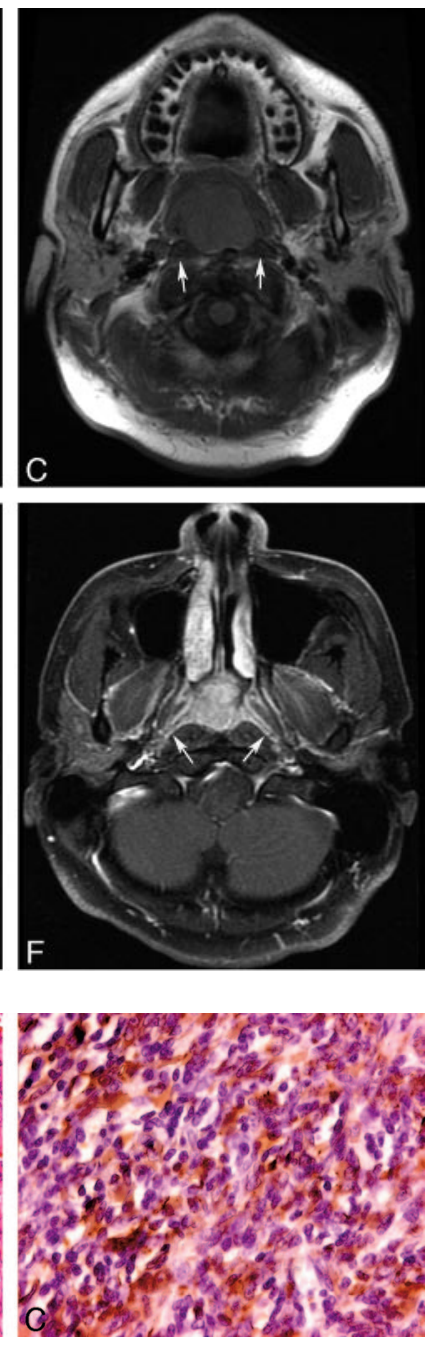

Fig 1. MR images of a 23-year-old man with a mass in the nasopharynx.

$A$, Sagittal gadolinium-enhanced fat-suppressed T1-weighted MR image reveals a well-demarcated tumor arising from the posterior wall of nasopharynx (arrow) with downward extension to oropharynx and compressing the soft palate and uvula (arrowhead).

$B$, Coronal gadolinium-enhanced fat-suppressed T1-weighted MR image shows that the tumor is confined within the nasopharynx and oropharynx without involvement of sphenopalatine foramen and pterygopalatine fossa on either side.

C, Axial T1-weighted image shows intermediate signal intensity of the tumor with lateral displacement of adjacent longus capitis muscles bilaterally (arrows).

$D$, Axial T2-STIR image at the same level as in $C$ shows high signal intensity of the tumor. An enlarged $1-\mathrm{cm}$ lymph node is seen in the right lateral retropharyngeal space (arrow).

$E$, Axial gadolinium-enhanced fat-suppressed T1-weighted MR image at the same level as in $C$ shows strong homogenous enhancement of the tumor.

$F$, Axial gadolinium-enhanced fat-suppressed T1-weighted MR image shows a normal mucosal pattern of the bilateral lateral pharyngeal recesses (arrows) without tumor involvement.

Fig 2. Histopathology of the tumor.

$A$, Features of spindle cell sarcoma are present $(H \& E$, original magnification $\times 200)$. $B$, The tumor cells are pleomorphic and have atypical mitoses (H\&E, original magnification $\times 400$ )

$C$, The tumor cells are strongly immunoreactive for desmin (Desmin, original magnification $\times 400$ ).

2. Fusconi M, Magliulo G, Della Rocca C, et al. Leiomyosarcoma of the sinonasal tract: a case report and literature review. Am J Otolaryngol 2002;23:108-11

3. Vogl TJ, Mack MG, Juergens M, et al. MR diagnosis of head and neck tumors: comparison of contrast enhancement with triple-dose gadodiamide and standard-dose gadopentetate dimeglumine in the same patients. AJR Am J Roent-

4. Fu YS, Perzin KH. Nonepithelial tumors of the nasal cavity, paranasal sinuses, and nasopharynx: a clinicopathologic study. IV. Smooth muscle tumors (leiomyoma, leiomyosarcoma). Cancer 1975;35:1300-08

5. Pimpinella RJ, Marquit B. Leiomyosarcoma of nose, nasopharynx and paranasal sinuses. Ann Otol Rhinol Laryngol 1965;74:623-30

6. Dobben GD. Leiomyosarcoma of the nasopharynx. AMA Arch Otolaryngol

7. Lippert BM, Godbersen GS, Luttges J, et al. Leiomyosarcoma of the nasal cavity: case report and literature review. ORL J Otorhinolaryngol Relat Spec

8. Ulrich CT, Feiz-Erfan I, Spetzler RF, et al. Sinonasal leiomyosarcoma: review of literature and case report. Laryngoscope 2005;115:2242-48

9. Kuruvilla A, Wenig BM, Humphrey DM, et al. Leiomyosarcoma of the sinonasal tract: a clinicopathologic study of nine cases. Arch Otolaryngol Head Neck 